International Journal of Computer Networks \& Communications (IJCNC) Vol.4, No.3, May 2012

\title{
EXTENDING THE CAPABILITY OF ENERGY DETECTOR FOR SENSING OF HETEROGENEOUS WIDEBAND SPECTRUM
}

\author{
Oluwatobi Olabiyi $^{1}$ and Annamalai Annamalai ${ }^{2}$ \\ Centre of Excellence for Communication Systems Technology Research \\ Department of Electrical and Computer Engineering \\ Prairie View A\&M University, Texas 77446 \\ lengr3os@gmail.com ${ }^{2}$ aaannamalai@pvamu.edu
}

\begin{abstract}
Reliable spectrum sensing is the very task upon which the entire operation of the cognitive radio rests. Energy detection is one of the solutions that have been proposed for enabling opportunistic spectrum access but its capability has limited sensitivity, no resolution and requires high sampling rate when employed in the detection of wideband heterogeneous spectrum. This article proposes a solution that can extend the capability of traditional energy detector to wideband sensing with improved performance. The solution involves the use of parallel detection circuitry with each tuned to different frequencies. The decision of each detection circuit can then be used to create a spectral map of the entire spectrum. This introduces some resolution component and improved sensitivity based on the number of parallel circuits and bandwidth of each detection circuit. Since the bandwidth of the incumbent signal is generally not known a priori, there is possibility that it spans across multiple detectors. The detection of such wideband signal can be achieved by either data or decision fusion of the results of each narrowband detector. We show that the use of parallel multi-channel detection can greatly enhance the detection of wideband signal compared to averaging while improving the resolution and sensitivity of energy detection of wideband spectrum. Further performance improvement can be achieved through the use of diversity reception on each narrow band detectors. Numerical results have been shown for representative cases and careful review of previous works reveals that these have never been considered in literature.
\end{abstract}

\section{KEYWORDS}

Energy Detectors, Heterogeneous Wideband Spectrum Sensing, Modified Periodogram, Cognitive Radios

\section{INTRODUCTION}

The opportunistic spectrum access (OSA) is a spectrum access system that facilitates the exploitation of local and instantaneous spectrum availability without having deleterious effect with the primary user [1]. That is, the spectrum access is accomplished without negotiating or consulting with the primary or licensed user. The OSA is the very foundation on which the success of cognitive radio transmission is built [2]-[6]. Hence, there is need for efficient spectrum detection techniques that can ensure cognitive radio transmission while protecting the incumbent transmissions. The main purpose of spectrum sensing is to become aware of the spatio-temporal electromagnetic environment by determining the frequencies occupied by the primary user [7]. Therefore, the performance of a spectrum sensing technique can be evaluated based on the following factors (i) the sensing latency (i.e. how long it takes to scan a particular spectrum and decide if cognitive transmission is possible); (ii) resolution (ability to detect hole within heterogeneous signals), (iii) sensitivity (ability to sense wideband spectrum within a particular period). Several methods of detecting spectrum holes including the matched filter 
International Journal of Computer Networks \& Communications (IJCNC) Vol.4, No.3, May 2012

detection, cyclostationary feature extraction, energy detection, wavelet detection and covariance detection have been proposed in the literature. Among these techniques energy detection is considered by many as the most versatile mainly due to the simplicity of its implementation, the non-coherent nature of its operation and its non-cognizance of incumbent signal to be detected.

The analysis of energy detection in the cognitive radio system has been considered in many articles. In [8]-[13] and related references, the detection of an unknown deterministic signal was formulated as a binary hypothesis testing problem. Here, the detection statistics is based on the Neyman-Pearson criterion and the performance is expressed in terms of false alarm and detection probability. These articles mostly focus on the reliability and accuracy of the spectrum sensing system. In [14]-[16] and related articles, the authors shifted their focus on sensing latency. The theory of quickest detection based on a statistical test to detect the change of distribution in observations as quickly as possible has been employed to minimize the transient time between the two states while guaranteeing certain false alarm probability. These methods employ well known algorithms like generalized likelihood ratio (GRL) test, windowed GRL, parallel cumulative sum (CUSUM) test and so on. Unfortunately, the energy detection methods in all the above mentioned literature have no resolution component. Also, its sensitivity is greatly hampered by the practical limitation of sampling rate of the analog-to-signal converter (ADC). All these factors limit the application of energy detector to only narrowband signal sensing and unsuitable for sensing in wideband heterogeneous environment. Although, frequency domain implementation like the (modified) periodogram can overcome the resolution problem, however, this technique is still limited by high ADC sampling rate requirement.

In recent articles [17]-[18], the detection of a wideband spectrum has been achieved by dividing the wideband spectrum into multiple channels using the theory of quickest detection. Authors in [17] considered a case when a single narrowband energy detector (capable of changing its reception parameters) is to sense multiple channels. The detector uses preset belief factor based on the past primary user activity to determine which channel to sense in the future. This approach has been shown to reduce the sensing time while guaranteeing certain false alarm rate compared to when the narrowband detector only focus on single channel. The authors in [18] later extended this analysis to the case of multiple narrowband detectors being used to sense multiple narrow band channels. Also, the number of channels is assumed to be more than the number of detectors. The authors followed similar analysis in [17] to arrive at the same conclusion that, using some believe factor, more spectrum holes can be harvested compared to focusing each detector on a particular narrowband at all times. The inherent assumption made in these two articles is that, the dynamic range of each detector spans the entire wide bandwidth while sensing only one narrowband per time. This assumption requires fast changes of the frequency of the local oscillator which imposes its own limitation on the practicality of this approach. More so, it heavily relies on the accurate knowledge of the distribution of primary user activities to reach optimality. Also, in a heterogeneous environment with combination of both wideband and narrowband signals, what happens when a wideband signal spans multiple band? It is not very obvious how to make sensing decision on multiple contiguous narrow bands as the choice of bands to be detected is random.

In this article, we propose an energy detector that overcomes these limitations by the use of parallel detection circuitry of varying contiguous centre frequency with each circuit tuned to a particular narrow bandwidth. Since the bandwidth of the incumbent signal is generally not known a priori, there is possibility that it spans across multiple detectors. In this case, we employ the use of traditional data and decision fusion to arrive at an overall decision over the wideband. While the data and decision fusion have been employed in earlier works [19]-[22] to improve the performance and reliability of energy detection with distributed cooperative spectrum sensing, their analysis are still limited to known narrow bandwidth. In contrast, here 
International Journal of Computer Networks \& Communications (IJCNC) Vol.4, No.3, May 2012

the arrangement allows us to introduce flexible resolution, improve the sensitivity, reduce the sensing time, overcome the practical limitation of the sampling rate of ADC and facilitate the

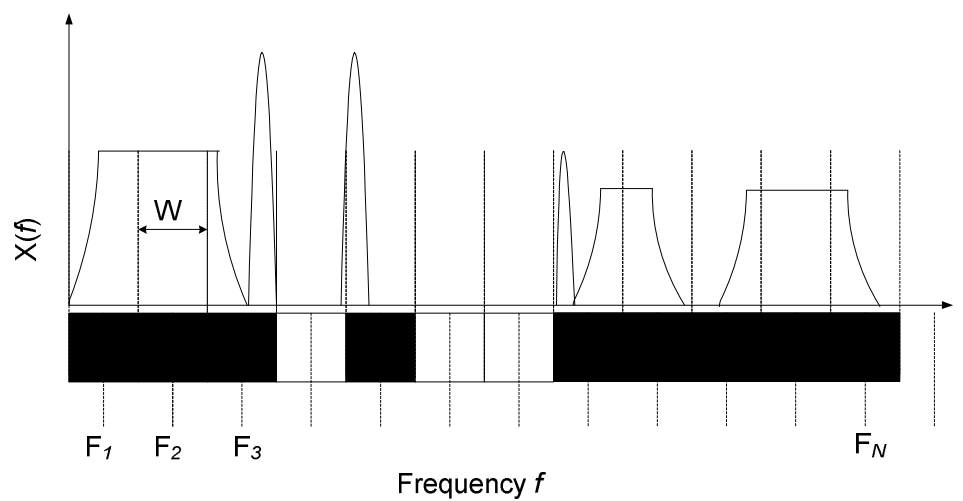

Fig. 1 Schematic diagram of parallel narrowband detectors (with different centre frequency $F_{i}$ and bandwidth $W_{i}$ ) combined to increase the sensitivity and resolution of traditional energy detector.

Sensing of heterogeneous wideband signal that might span a spectrum covered by multiple contiguous narrowband energy detectors. Furthermore, the effect of diversity reception by each NB detector on the overall detection performance is investigated. Numerical results indicates that channelization using multiple narrow band (NB) energy detector improves the overall sensing performance over traditional wideband detector. Also, the combination of channelization and diversity reception may reduce the number of NB detector that is required to achieve certain performance target. Careful review of previous work reveals that such practical application of energy detection to sensing of wideband heterogeneous signal detection has never been presented in the literature

The remainder of this article is organized as follows. The system model and the analysis of parallel multi-channel detection for wideband heterogeneous spectrum sensing is discussed in Section 2. Section 3 introduces an additional performance improvement through the use of diversity reception. Selected numerical results are provided in Section 4, while Section 5 summarizes the key results.

\section{SYSTEM MODEL}

Consider a wideband heterogeneous spectrum of bandwidth, $W_{T}$ which covers the spectrum range of $N$ narrowband (NB) energy detectors as shown in Fig. 1 . The choice of $N$ depends on the resolution required with higher $N$ leading to more detection of white spaces as could be seen in Fig 1. However, large $N$ value increases the complexity of detection circuitry. The centre frequency and bandwidth of the detector's receiver of $i^{\text {th }} \mathrm{NB}$ spectrum is given by $F_{i}$ and $W_{i}$ respectively. Therefore, the total bandwidth of parallel multi-channel detector (PMD) is given by $W_{T}=\sum_{i=1}^{N} W_{i}$. The detection of the existence of an unknown deterministic signal $s_{i}(t)$ within the bandwidth $W_{i}$ can then be analysed as normal energy detection problem ([8]-[11] and related references) which is a binary hypothesis test as shown in [8, eq. (1)] via,

$$
y_{i}(t)=\left\{\begin{array}{cc}
n_{i}(t) & : H_{0} \\
h s_{i}(t)+n_{i}(t) & : H_{1}
\end{array}\right.
$$

where $s_{i}(t)$ is the unknown deterministic signal waveform received by detector $i, n_{i}(t)$ is the noise waveform (white Gaussian random process), $H_{0}$ is the hypothesis 0 (i.e., no $s_{i}(t)$ present) 
International Journal of Computer Networks \& Communications (IJCNC) Vol.4, No.3, May 2012

and $H_{1}$ is the hypothesis 1 (i.e., $s_{i}(t)$ present). Therefore, a sample from noise process $n_{i}$ is a Gaussian random variable with zero mean and $N_{01} W_{i}$ variance; $n_{i}=N\left(0, N_{01} W_{i}\right)$ [9], where $N_{01}$ is one sided noise power spectral density, $W_{i}$ is one-sided bandwidth. The decision statistics, $Y_{i}$ according to [9] under the null hypothesis, $H_{0}$ is a square sum of $2 u_{i}$ Gaussian random variables of $N(0,1)$, and thus follows $\chi_{2 u_{i}}^{2}$ where $u_{i}=T W_{i}$ is the time-bandwidth product which is the number of samples considered for detection at detector $i . Y_{i}$ under the alternative hypothesis, $H_{1}$ is $\chi_{2 u_{i}}^{2}\left(2 \gamma_{i}\right)$, where $2 \gamma_{i}$ is the non-centrality parameter and $\gamma_{i}$ is the received SNR of the signal detected by the $i^{\text {th }}$ detector. Thus the probability of detection $\left(P_{d_{0}}^{(i)}\right)$ and the probability of false alarm $\left(P_{f_{0}}^{(i)}\right)$ of an unknown deterministic signal $s_{i}(t)$ in additive white Gaussian noise is given by [9].

$$
\begin{gathered}
P_{d_{0}}^{(i)}=Q_{u_{i}}\left(\sqrt{2 \gamma_{i}}, \sqrt{\lambda}\right) \\
P_{f_{0}}^{(i)}=\Gamma\left(u_{i}, \frac{\lambda}{2}\right) / \Gamma\left(u_{i}\right)
\end{gathered}
$$

where $\gamma$ in (2) corresponds to the SNR of received signal by $i^{\text {th }}$ detector, $\lambda$ denotes the threshold of the detector, $Q_{u}(. .$.$) is the generalized Marcum Q-function of order u$, and $\Gamma(.,$.$) is the upper$ incomplete gamma function which is defined by the integral $\Gamma(a, x)=\int_{x}^{\infty} t^{a-1} e^{-t} d t$.

From (2) and (3), we can observe the false alarm probability is not affected by the strength of the received signal as (3) is not dependent on $\gamma$ term. However, (2) depends on the received signal-to-noise ratio (SNR) which is random in fading channels. Therefore, there is need to average (2) over the distribution of SNR in specific fading environment.

The average detection probability over an arbitrary fading channel with probability density function (PDF) $f_{\gamma}(\gamma)$ can be evaluated by

$$
\overline{P_{d_{0} \text { Gen }}^{(i)}}=\int_{0}^{\infty} Q_{u_{i}}\left(\sqrt{2 \gamma_{i}}, \sqrt{\lambda}\right) f_{\gamma}\left(\gamma_{i}\right) d \gamma_{i}
$$

Using the canonical series representation of the Marcum Q-function in [23], and taking advantage of the Laplace differentiation identity, the average detection probability over a generalized fading channel can be expressed as [9]

$$
\begin{aligned}
\overline{P_{d_{0} \text { Gen }}^{(i)}} & =1-\sum_{k=0}^{\infty} \frac{(-1)^{k}}{k !} \frac{G\left(u_{i}+k, \frac{\lambda}{2}\right)}{\Gamma\left(u_{i}+k\right)} \int_{0}^{\infty} \gamma_{i}^{k} e^{-\gamma_{i}} f_{\gamma_{i}}\left(\gamma_{i}\right) d \gamma \\
& =1-\left.\sum_{k=0}^{\infty} \frac{(-1)^{k}}{k !} \frac{G\left(u_{i}+k, \frac{\lambda}{2}\right)}{\Gamma\left(u_{i}+k\right)} \phi_{\gamma_{i}}^{(k)}(s)\right|_{s=1}
\end{aligned}
$$

where $\phi_{\gamma}^{(k)}(s)=\partial^{k} \phi_{\gamma}(s) / \partial s^{k}$ and $\phi_{\gamma_{i}}(s)=\int_{0}^{\infty} e^{-s \gamma_{i}} f_{\gamma_{i}}\left(\gamma_{i}\right) d \gamma$ is the MGF of SNR of stochastic fading channel, and $G(.,$.$) is the lower incomplete gamma function which is defined by$ $G(a, x)=\int_{0}^{x} t^{a-1} e^{-t} d t$. The convergence of this infinite series has been well treated in [9] and it's been shown that only few terms are required for four-digit accuracy. The $k$ th order derivative, $\phi_{\gamma}^{(k)}(s)$ for common fade distributions have been derived in Appendix A and can be employed in computing the average detection probability in a specified fading channel. For instance, the average detection probability in Nakagami-m and Rice channels can be computed efficiently using (6) and (7), respectively: 
International Journal of Computer Networks \& Communications (IJCNC) Vol.4, No.3, May 2012

$$
\begin{gathered}
\bar{P}_{d \text { Nak }}=1-\sum_{k=0}^{\infty} \frac{1}{k !} \frac{G\left(u_{i}+k, \frac{\lambda}{2}\right)}{\Gamma\left(u_{i}+k\right)} \frac{\Omega^{k} m^{m} \Gamma(m+k)}{(m+\Omega)^{m+k} \Gamma(m)} \\
\bar{P}_{d R i c}=1-\sum_{k=0}^{\infty} \frac{1}{k !} \frac{G\left(u_{i}+k, \frac{\lambda}{2}\right)}{\Gamma\left(u_{i}+k\right)} \frac{\Omega^{k}(k !)^{2}(1+K)}{(1+K+\Omega)^{1+k}} \exp \left(\frac{-K \Omega}{1+K+\Omega}\right) \sum_{j=0}^{k} \frac{1}{(j !)^{2}(k-1) !}\left(\frac{K(1+K)}{1+K+\Omega}\right)^{j}
\end{gathered}
$$

The decision of each energy detection circuitry can then be used to create spectral map of the entire spectrum as shown in Fig 1. Also, if single overall detection decision is required across the bands covered by multiple detectors, due to the presence of a wideband primary user (PU) signal or wideband cognitive user transmission, the decision statistics of each detector can be combined using the data or decision fusion. This will be considered next.

\subsection{Data Fusion}

The data fusion uses the statistics of the decision variable to make the overall decision. Some of the known statistics include minimum, maximum and average. The performance of the each of these statistics shall be considered next.

\section{Case 1: Minimum Selection}

Here, the detection decision is made only when the detector with minimum decision variable exceed the detection threshold i.e. $Y=\min \left(Y_{1}, Y_{2}, \ldots Y_{N}\right)$.

The detection and false alarm probabilities are then respectively given by

$$
\begin{aligned}
P_{d}^{T} & =\operatorname{Pr}\left[\min \left(Y_{1}, Y_{2}, \ldots Y_{N}\right)>\lambda \mid H_{1}\right] \\
& =\prod_{i=1}^{N}\left\{\operatorname{Pr}\left[Y_{i}>\lambda \mid H_{1}\right]\right\} \\
& =\prod_{i=1}^{N}\left[Q_{u_{i}}\left(\sqrt{2 \gamma_{i}}, \sqrt{\lambda}\right)\right]
\end{aligned}
$$

and

$$
\begin{aligned}
P_{f}^{T} & =\operatorname{Pr}\left[\min \left(Y_{1}, Y_{2}, \ldots Y_{N}\right)>\lambda \mid H_{0}\right] \\
& =\prod_{i=1}^{N}\left\{\operatorname{Pr}\left[Y_{i}>\lambda \mid H_{0}\right]\right\} \\
& =\prod_{i=1}^{N}\left[\Gamma\left(u_{i}, \frac{\lambda}{2}\right) / \Gamma\left(u_{i}\right)\right]
\end{aligned}
$$

The corresponding average detection probability over the generalized fading channel is then given by

$$
{\overline{P_{d_{\text {mim Gen }}}}}=\prod_{i=1}^{N}\left[1-\left.\sum_{k=0}^{\infty} \frac{(-1)^{k}}{k !} \frac{G\left(u_{i}+k, \frac{\lambda}{2}\right)}{\Gamma\left(u_{i}+k\right)} \phi_{\gamma_{i}}^{(k)}(s)\right|_{s=1}\right]
$$

\section{Case2: Averaging}

In this case, a global decision can be made by considering the average of the entire local decisions variables i.e. $Y=\left(Y_{1}+Y_{2}+\ldots+Y_{N}\right) / N$. Interestingly, averaging does not affect the mean of a statistic but increases the degree of freedom by order $N$ and reduces the variance of a statistics by the factor of $N$. Therefore, the detection and false alarm probability are respectively given by

$$
\begin{aligned}
P_{d}^{T} & =\operatorname{Pr}\left[\left(Y_{1}+Y_{2}+\ldots Y_{N}\right) / N>\lambda \mid H_{1}\right] \\
& =Q_{u}\left(\sqrt{2 \gamma_{T}}, \sqrt{\lambda}\right)
\end{aligned}
$$


and

$$
\begin{aligned}
P_{f}^{T} & =\operatorname{Pr}\left[\left(Y_{1}+Y_{2}+\ldots Y_{N}\right) / N>\lambda \mid H_{0}\right] \\
& =\Gamma\left(u, \frac{\lambda}{2}\right) / \Gamma(u)
\end{aligned}
$$

where $u=\sum_{i=1}^{N} u_{i}$ and $\gamma_{T}=\sum_{i=1}^{N} \gamma_{i}$ is the received SNR of the wideband band signal over the bandwidth $W=\sum_{i=1}^{N} W_{i}$. The result of (11) and (12) is the same as the conventional energy detector over a wideband [9] (i.e., the average detection probability is given by (5) but replacing $u_{i}$ by $u$ ). The advantages of the channelization of sensing bandwidth include removing the burden of high sampling frequency rate for ADCs, and quicker sensing time (due to "parallel" processing) but at the expense of increased hardware costs.

\section{Case3: Maximum Selection}

In this case, the NB detector with maximum or peak detection decision variable can be used to make global detection decision i.e. $Y=\max \left(Y_{1}, Y_{2}, \ldots Y_{N}\right)$. Therefore the detection and false alarm probabilities are given by

$$
\begin{aligned}
P_{d}^{T} & =\operatorname{Pr}\left[\max \left(Y_{1}, Y_{2}, \ldots Y_{N}\right)>\lambda \mid H_{1}\right] \\
& =1-\prod_{i=1}^{N}\left\{1-\operatorname{Pr}\left[Y_{i}>\lambda \mid H_{1}\right]\right\} \\
& =1-\prod_{i=1}^{N}\left[1-Q_{u_{i}}\left(\sqrt{2 \gamma_{i}}, \sqrt{\lambda}\right)\right]
\end{aligned}
$$

and

$$
\begin{aligned}
P_{f}^{T} & =\operatorname{Pr}\left[\max \left(Y_{1}, Y_{2}, \ldots Y_{N}\right)>\lambda \mid H_{0}\right] \\
& =1-\prod_{i=1}^{N}\left\{1-\operatorname{Pr}\left[Y_{i}>\lambda \mid H_{0}\right]\right\} \\
& =1-\prod_{i=1}^{N}\left[G\left(u_{i}, \frac{\lambda}{2}\right)\right]
\end{aligned}
$$

The corresponding average detection probability over the generalized fading channel is then given by

$$
{\overline{P_{d_{\text {mim } G e n}}}}=1-\prod_{i=1}^{N}\left[\left.\sum_{k=0}^{\infty} \frac{(-1)^{k}}{k !} \frac{G\left(u_{i}+k, \frac{\lambda}{2}\right)}{\Gamma\left(u_{i}+k\right)} \phi_{\gamma_{i}}^{(k)}(s)\right|_{s=1}\right]
$$

\subsection{Decision Fusion}

Another method of making detection decision of wideband signal is to allow each NB detector make local decision and then fuse the decisions to make final global decision. This is similar to decision fusion that has been well treated in literature. The main difference here, is that there is no reporting error as all the decisions are locally combined.

For a $N$ NB detectors, assuming $k$-out-of- $N$ decision fusion rule i.e. a decision is reached once $k$ out of $N$ NB detector agrees, the effective detection and false alarm probabilities is generally given by

$$
P_{\chi}^{T}=\sum_{i=k \ldots N} \prod_{j=1}^{i} P_{\chi}^{(j)} \prod_{j=i+1}^{N}\left(1-P_{\chi}^{(j)}\right)
$$

where $\left(\chi=f^{\prime}\right)$ corresponds to the false alarm probability and $\left(\chi={ }^{\prime} d^{\prime}\right)$ corresponds to the detection probabilities.

For the special case of $k=1$ (this corresponds to ' $O R$ ' decision rule), (13) becomes

$$
P_{\chi}^{T}(k=1)=1-\prod_{i=1}^{N}\left(1-P_{\chi}^{(i)}\right)
$$

which is similar to the expression in (13) and (14). 
International Journal of Computer Networks \& Communications (IJCNC) Vol.4, No.3, May 2012

Also for the special cases of 'AND' $(k=N)$ and Majority $(k=\lceil N / 2\rceil)$ decision rules, we obtain from (13):

$$
\begin{gathered}
P_{\chi}^{T}(k=N)=\prod_{i=1}^{N} P_{\chi}^{(i)} \\
P_{\chi}^{T}(k=\lceil N / 2\rceil)=\sum_{i=[N / 2\rceil \ldots N} \prod_{j=1}^{i}\left(P_{\chi}^{(i)}\right) \prod_{j=i+1}^{N}\left(1-P_{\chi}^{(i)}\right)
\end{gathered}
$$

It is worth to mention that for independent and identical statistics, both "OR" and "AND" decision fusion rules respectively give the same result as the minimum and maximum statistics of data fusion.

\section{PERFoRMANCE IMPROVEMENT WITH DIVERSITY RECEPTION}

Additional detection performance improvement can be achieve if each NB detector are capable of diversity reception. Diversity-enhanced energy detection for NB detector has been shown in [9]- [11] to be beneficial by reducing the miss-detection probability in the order of magnitude of $L$, where $L$ is the number of diversity branches. Since diversity reception improves the performance of each NB detector, better detection performance of the wideband signal is expected. Most of the diversity detection techniques can be applied to energy detector, however, we shall limit the analysis in this article to maximum ratio combining (MRC) and square law combining (SLC).

\subsection{Pre-detection Diversity Combining - Maximum Ratio Combining (MRC)}

In the MRC scheme, the signals from $L$ independent diversity branches are being combined before sampling and therefore the MRC output SNR is simply the sum of the instantaneous SNRs from all diversity branches, i.e., $\gamma_{M R C}=\sum_{l=1}^{L} \gamma_{l}$. Also, the decision statistics $Y_{M R C}$ under $H_{1}$ follows $\chi_{2 u}^{2}(\epsilon)$ non-centrality parameter $\sum_{l=1}^{L} 2 \gamma_{l}$ while the decision statistics $Y_{M R C}$ follows $\chi_{2 u}^{2}$ under $H_{0}$. Hence, the conditional detection probability of the $i$ th NB detector can be evaluated as

$$
P_{d, M R C}^{(i)}=Q_{u}\left(\sqrt{2 \gamma_{i M R C}}, \sqrt{\lambda}\right)=1-\sum_{k=0}^{\infty} \frac{\gamma_{i M R C}^{k} e^{-\gamma_{M R C}}}{k !} \frac{G\left(u_{i}+k, \frac{\lambda}{2}\right)}{\Gamma\left(u_{i}+k\right)},
$$

while the false alarm probability is identical to Eq. (3). The average detection probability with MRC diversity receiver can be evaluated in a similar fashion to our development leading to Eq. (5), but with an exception that $\phi_{\gamma_{\text {iMRC }}}^{(k)}(s)$ is now required. The $\phi_{\gamma_{\text {MRCC }}}^{(k)}(s)$ for the special case of i.i.d fading statistics can be obtained in Appendix A. Hence, the corresponding average detection probabilities in Nakagami- $m$ and Rice fading are shown in Equations (21) and (22), respectively:

$$
\begin{gathered}
{\overline{P_{d}^{(i)}}}_{M R C-N a k}=1-\sum_{k=0}^{\infty} \frac{1}{k !} \frac{G\left(u_{i}+k, \frac{\lambda}{2}\right)}{\Gamma\left(u_{i}+k\right)} \frac{\Omega^{k} m^{L m} \Gamma(L m+k)}{(m+\Omega)^{L m+k} \Gamma(L m)} \\
{\overline{P_{d}^{(i)}}}_{M R C-R i C}=1-\sum_{k=0}^{\infty} \frac{1}{k !} \frac{G\left(u_{i}+k, \frac{\lambda}{2}\right)}{\Gamma\left(u_{i}+k\right)} \frac{\Omega^{k} k !(L+k-1) !(1+K)^{L}}{(1+K+\Omega)^{L+k}} \exp \left(\frac{-K L \Omega}{1+K+\Omega}\right) \sum_{j=0}^{k} \frac{1}{j !(L+j-1) !(k-j) !}\left(\frac{K L(1+K)}{1+K+\Omega}\right)^{j}
\end{gathered}
$$

For the more general case of i.n.d branch statistics, we obtain the MGF of MRC output SNR as $\phi_{\gamma_{\text {MRRC }}}(s)=\prod_{l=1}^{L} \phi_{i l}(s)$. In this case, we can derive a closed-form expression for $\phi_{\gamma_{\text {MRRC }}}^{(k)}(s)$ using the Leibniz differentiation rule, viz.,

$$
\phi_{\gamma_{\text {MRCC }}}^{(k)}(s)=\sum_{n_{1}=0}^{k} \sum_{n_{2}=0}^{n_{1}} \cdots \sum_{n_{L-1}=0}^{n_{L-2}}\left(\begin{array}{c}
k \\
n_{1}
\end{array}\right)\left(\begin{array}{c}
n_{1} \\
n_{2}
\end{array}\right) \cdots\left(\begin{array}{c}
n_{L-2} \\
n_{L-1}
\end{array}\right) \phi_{i 1}^{\left(k-n_{1}\right)}(s) \phi_{i 2}^{\left(n_{1}-n_{2}\right)}(s) \cdots \phi_{i L}^{\left(n_{L-1}\right)}(s)
$$


International Journal of Computer Networks \& Communications (IJCNC) Vol.4, No.3, May 2012

The corresponding average detection probability can be easily obtained by substituting Eq. (23) into Eq. (5). It is also important to highlight that Eq. (23) circumvents the need for partial fraction expansion of the MGF, and can be easily implemented on most mathematical software packages such as MATHEMATICA and MAPLE.

The effect of diversity can be easily observed from the high-SNR approximation of Eq. (21) by expressing it in the form

$$
\bar{P}_{d}=1-P_{m} \Omega^{-g_{m}}
$$

where $P_{m}=\sum_{k=0}^{\infty} \frac{1}{k !} \frac{G\left(u+k, \frac{\lambda}{2}\right)}{\Gamma(u+k)} \frac{m^{L m} \Gamma(L m+k)}{\Gamma(L m)}$ is the auxiliary miss-detection probability and $g_{m}=L m$ is the diversity gain. It can be observed that as diversity order $L$ increases, the diversity gain $g_{m}$ increases and therefore the miss-detection probability decreases, which ultimately improves the energy detection performance.

\subsection{Post-detection Diversity Combining - Square Law Combining (SLC)}

In the square-law combining receiver, the output decision is combined after sampling. The decision statistics $Y_{S L C}$ is thus the sum of $L$ i.i.d $\chi_{2 u}^{2}$ under $H_{0}$ and the sum of $L$ i.i.d $\chi_{2 u}^{2}(\epsilon)$ under $H_{1}$ as [9]

$$
Y_{S L C}=\sum_{l=1}^{L} Y_{l}=\left\{\begin{array}{cl}
\chi_{2 L u}^{2} & : H_{0} \\
\chi_{2 L u}^{2}\left(e_{S L C}\right) & : H_{1}
\end{array}\right.
$$

where $\epsilon_{S L C}=\sum_{l=1}^{L} \epsilon_{l}=\sum_{l=1}^{L} 2 \gamma_{l}=2 \gamma_{S L C}$. Hence, the false alarm and the detection probabilities in AWGN are given by

$$
\begin{gathered}
P_{f, S L C}^{(i)}=\frac{\Gamma\left(L u_{i}, \frac{\lambda}{2}\right)}{\Gamma\left(L u_{i}\right)} \\
P_{d, S L C}^{(i)}=Q_{L u_{i}}\left(\sqrt{2 \gamma_{i S L C}}, \sqrt{\lambda}\right)=1-\sum_{k=0}^{\infty} \frac{\gamma_{i S L C}^{k} e^{-\gamma_{S L C}}}{k !} \frac{G\left(L u_{i}+k, \frac{\lambda}{2}\right)}{\Gamma\left(L u_{i}+k\right)}
\end{gathered}
$$

Then, the average detection probability with SLC diversity over a generalized fading channel can be computed as

$$
{\overline{P_{d}^{(i)} G e n}}=1-\left.\sum_{k=0}^{\infty} \frac{(-1)^{k}}{k !} \frac{G\left(L u_{i}+k, \frac{\lambda}{2}\right)}{\Gamma\left(L u_{i}+k\right)} \phi_{\gamma_{S L C}}^{(k)}(s)\right|_{s=1}
$$

where $\phi_{\gamma_{\text {SLCC }}}(s)=\phi_{\gamma_{\text {MRC }}}(s)=\prod_{l=1}^{L} \phi_{i l}(s)$ since $\gamma_{i S L C}=\gamma_{i M R C}$. For instance, the $k$-th derivative of the MGF of MRC output SNR provided in Appendix A can be directly used in Eq. (28) to compute the average detection probabilities with i.i.d $L$-branch SLC diversity in a myriad of fading environments. The SLC diversity detector provides good alternative to MRC but with reduced detection performance due to increase in the degree of freedom and therefore higher false alarm probability.

\section{Computational Results}

In this section, we show through numerical analysis the capability of our proposed framework. In Fig. 2 - 5, we consider the sensing of a wideband signal of arbitrary bandwidth $W_{T}$ from $2 u$ samples. In order to keep the total number of samples constant, we divide it equally among the parallel detectors. First, we show the effect of number of parallel multi-channel detectors on the detection performance. Fig. 2 illustrates the detection performance of peak, average and minimum statistics for $N=2 \mathrm{NB}$ detectors. The figure shows that at constant threshold $\lambda=15 \mathrm{~dB}$, the minimum detector gives the worst detection performance (i.e. least protection to the PU signal) while the choice of either peak or average depends on the average SNR. It is worth to 
International Journal of Computer Networks \& Communications (IJCNC) Vol.4, No.3, May 2012

note that while the minimum detector has the least detection performance; it also has the least false alarm probability (which is desirable for an energy detector).

Fig. 3 shows the detection performance as the number of narrowband detectors increases. Similar trend as in Fig. 2 was observed. In addition, it shows that increasing the number of detectors (more resolution) worsens the performance of the minimum detector while it improves the performance of the peak detector. However, the average statistics is not affected by variation in number of NB detector because its performance relies solely on the total number of samples, $2 u$ which remains constant.

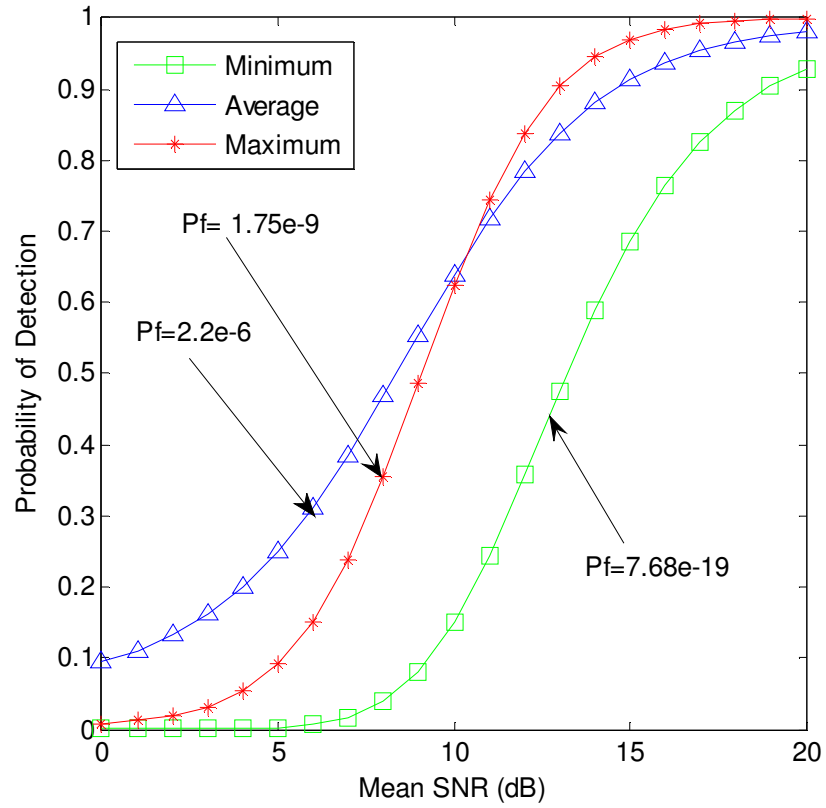

Fig. 2 Detection probability of wideband sensing with $N=2$ NB detectors over a Rice fading channel with $K=3$, total $u=10$, and $\lambda=15 \mathrm{~dB}$.

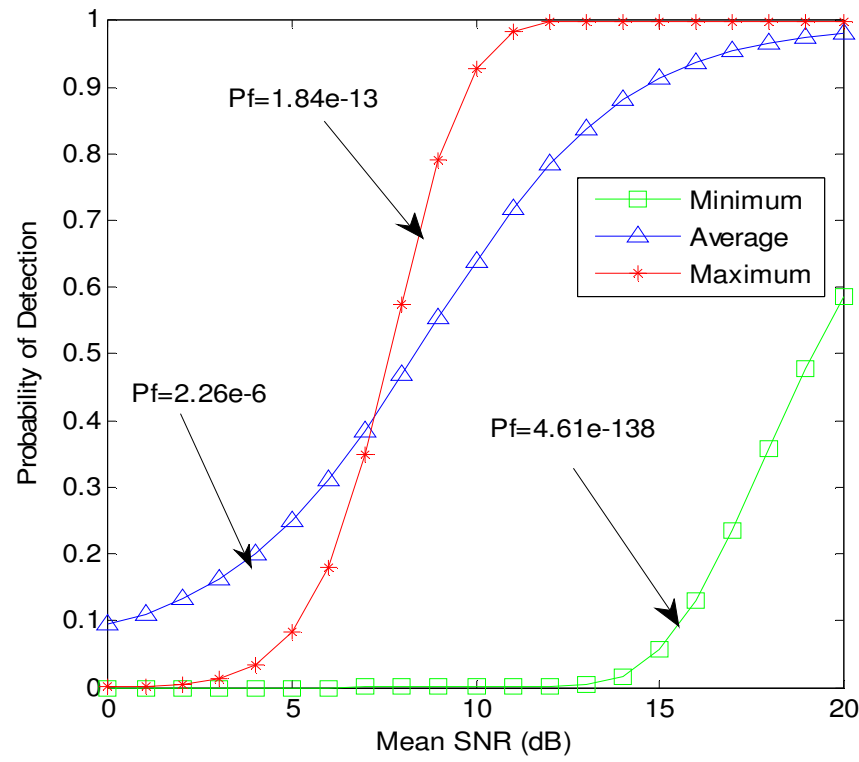

Fig. 3 Detection probability of wideband sensing with $N=10 \mathrm{NB}$ detectors over a Rice fading channel, $K=3$, total $u=10$, and $\lambda=15 \mathrm{~dB}$. 
It is important to also point out that with increasing number of narrow band detector, the performance of peak detector becomes better than that of the average detector at lower SNR values. Interestingly, Fig. 4 uses the NP criterion to differentiate the overall performance. Here, the false alarm probability is kept constant across the statistics which implies that the threshold can vary from one statistics to the other. The figure indicates that overall, the maximum selection gives better performance than the minimum selection with both giving better performance than the averaging (same as normal energy detection). Interesting to note here is that, the minimum detector which gives the least protection to the PU signal has the better overall detection performance than the traditional energy detector. Therefore, the use of minimum detector can be particularly useful in the unlicensed band where the PU signal protection requirement is minimal. In the same manner the application of peak detector will be more suitable for military application where PU protection is of priority.

In Fig. 5, the decision fusion of four NB detectors is considered and compared with the average energy detector using the complementary ROC curves (i.e., the average probability of miss detection, $\overline{P_{m}}=1-\overline{P_{d}}$ versus the false alarm probability $P_{f}$ ). It shows that all of the decision fusion rules give better performance than the simple averaging. This is in agreement with Fig. 4 since the OR rule $(k=1)$ corresponds to the peak detector and the 'AND' rule $(k=N)$ corresponds to the minimum detector. However, the choice of decision fusion rule depends on the system parameters and channel condition. It can be observed that the 'OR' rules has the best performance at high $P_{f}$ while the 'AND' rule $(k=N)$ is more appropriate at very low (small) $P_{f}$

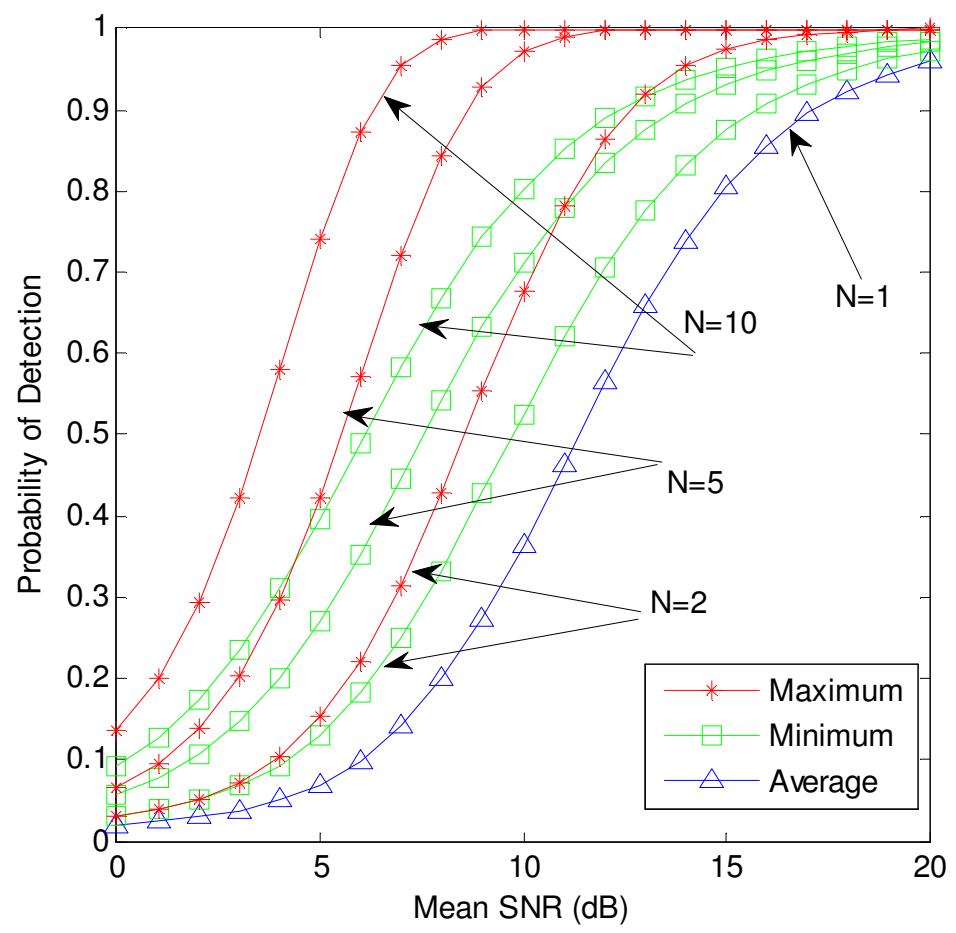

Fig. 4 Performance of wideband sensing with different number of NB detectors, $N=\{2,5,10\}$ over a Rice multipath fading channel, with $K=3$, total $u=10$ and $P_{f}=0.01$. 


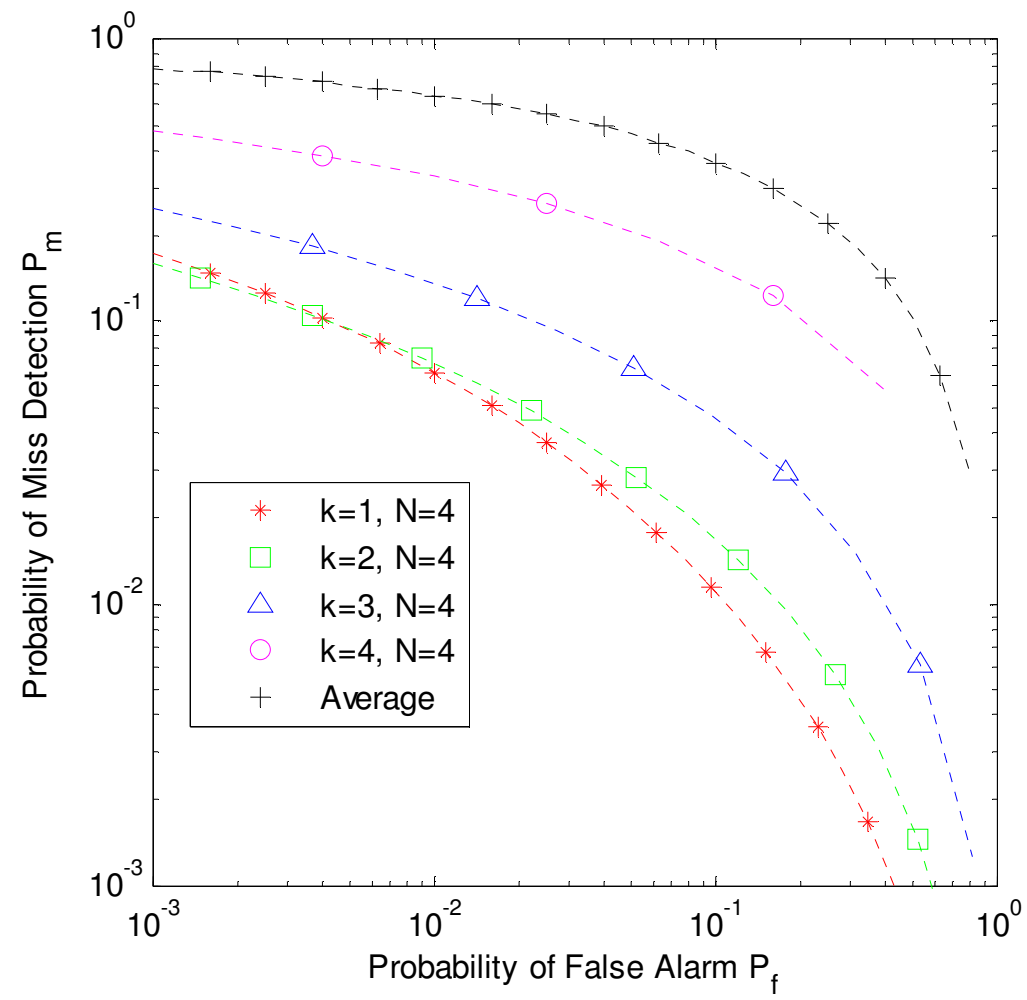

Fig. 5 Complementary ROC curves for $k$-out-of- $N$ decision fusion schemes of $N=4 \mathrm{NB}$ detectors over a Rice channel with $K=3$, total $u=10$, and mean SNR $=10 \mathrm{~dB}$.

Fig. 6 illustrates the effect of channel fading severity on the decision fusion scheme. In the overall, the performance of this scheme is improved with reduced fading severity (increasing $m$ values) for all values of $k$. However, further inspection reveals that, at poor channel condition, $k=1$ ('OR' rule) gives the best performance while $k=N$ ('AND' rule) giving the worst performance. Also, as the channel condition becomes better, higher values of $k$ gives better performance. This is in line with an intuitive expectation because, poor channel condition requires agreement of all the detectors to declare a wideband channel as vacant and offer maximum protection of the PU signal. However, as the channel condition becomes better, agreement of all the NB detector is not necessarily required to guarantee maximum protection to the primary user. In fact, at very good channel condition, for specified false alarm probability, the 'AND' rule might be sufficient enough. In this case, vacancy declaration by only one NB detector can be assumed for the entire wideband.

Fig. 7 depicts the performance of diversity-enhanced wideband energy detectors with MRC diversity scheme over the Rice fading channel. Here, the peak detector (maximum statistics or 1 -out of- $N$ rule) is used for the overall statistics of the wideband detection. The figure indicates that, increasing the number of diversity branches improves the overall detection of wideband signal for specific number of NB detectors. Although not shown, similar trend is observed for other statistics.

With a high impact from the introduction of diversity detection, it will be interesting to investigate whether it is more advantageous to increase the number of diversity or the number NB detectors in order to achieve a specified performance target. Answer to this question is of uttermost relevance in the implementation of wideband signal detectors and will be considered next. 
International Journal of Computer Networks \& Communications (IJCNC) Vol.4, No.3, May 2012

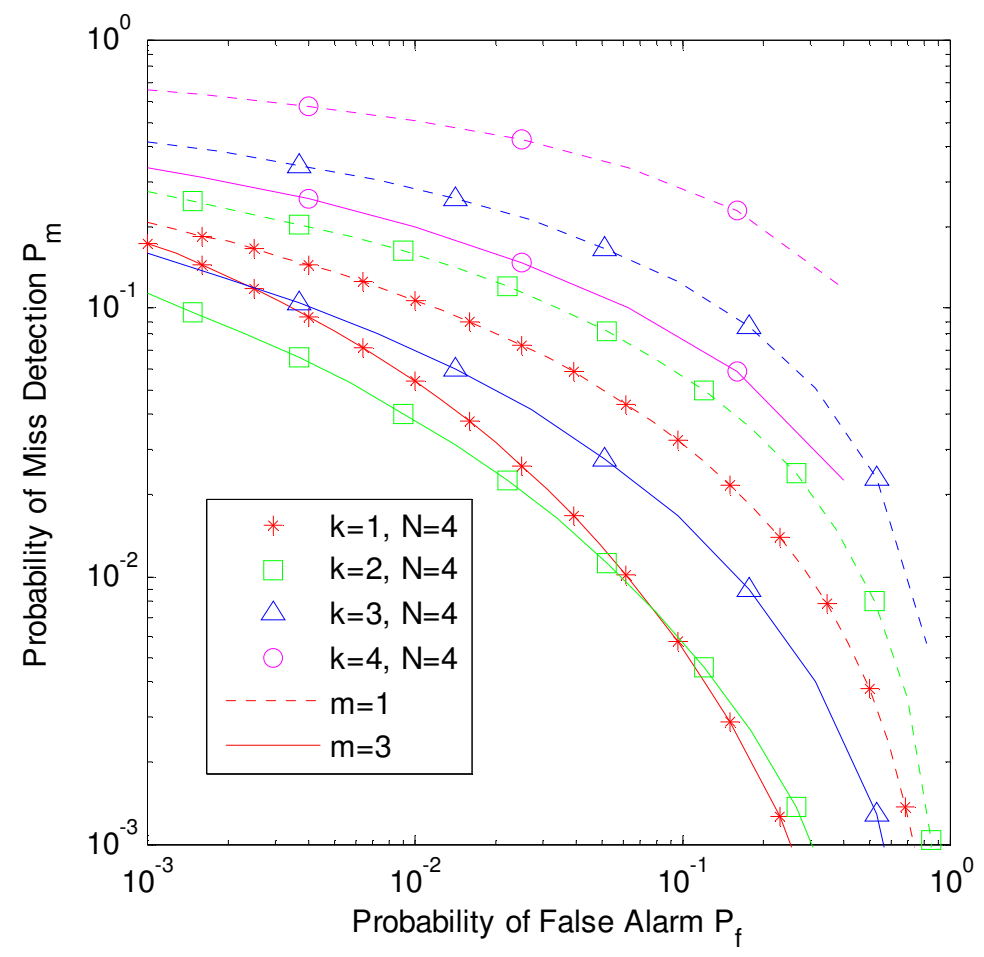

Fig. 6 Complementary ROC curves for $k$-out-of- $N$ decision fusion schemes of $N=4$ NB detectors over a Nakagami-m channel with $m=\{1,3\}$, total $u=10$, and mean SNR $=10 \mathrm{~dB}$.

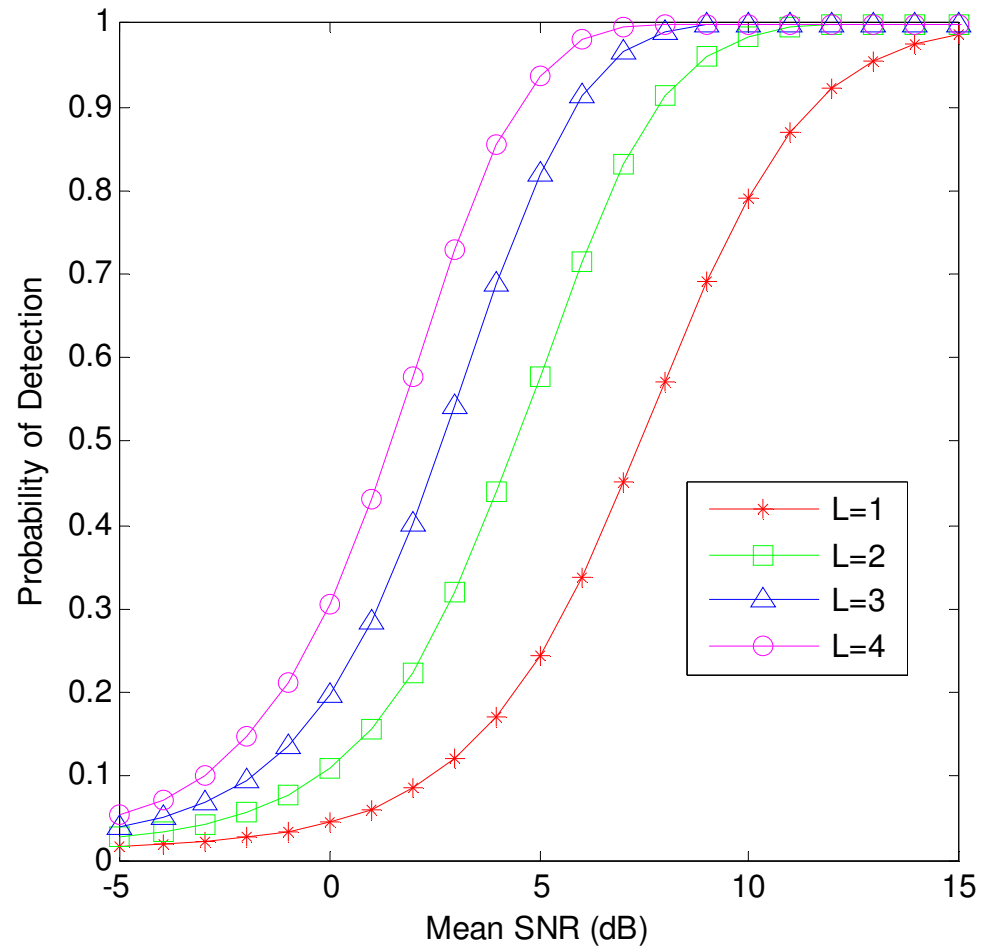

Fig. 7 Performance of wideband sensing using 2-NB peak detector with MRC diversity over a Rice $(K=3)$ multipath fading channel, with $L=\{1,2,3,4\}$, total $u=10$ and $P_{f}=0.01$. 
Fig. 8 shows independently the benefits of increasing both the number of NB energy detectors and diversity branches. The base configuration here is a wideband signal detector with $N=2 \mathrm{NB}$ detectors with no diversity reception. For the same bandwidth, the number of NB detector is double $(N=4)$ with no diversity (i.e. $L=1)$. In another arrangement, the number of diversity branches (with MRC scheme) is also doubled $(L=2)$ while keeping $N=2$. The figure shows that the detection improvement caused by increasing the diversity branches is more than the effect of channelization. Similar trend is observed when the factor is increased to 5. In fact, the gap in the performance increases as the order increases. Therefore, the $N L$ product can be used to ensure balanced compromise between the diversity order and channelization (i.e. number of NB detectors) during the implementation of wideband signal detectors.

Lastly, the performance of SLC and MRC diversity schemes is compared in Fig. 9. The figure indicates that as the diversity order $L$ increases, the performance gap between the MRC and SLC schemes widens with MRC scheme giving better performance across all range of SNR. This is because, since SLC decision is made after the signal from each diversity branch is sampled separately, the additional increase in the degree of freedom ultimately leads to increased false alarm probability which reduces the overall detection performance. However, it is worth to mention that since the diversity combination in SLC scheme is not coherent, it is more feasible to implement than the MRC scheme where all the signals have to be coherently combined before sampling. Therefore, SLC scheme is a recommended practical alternative to the MRC scheme.

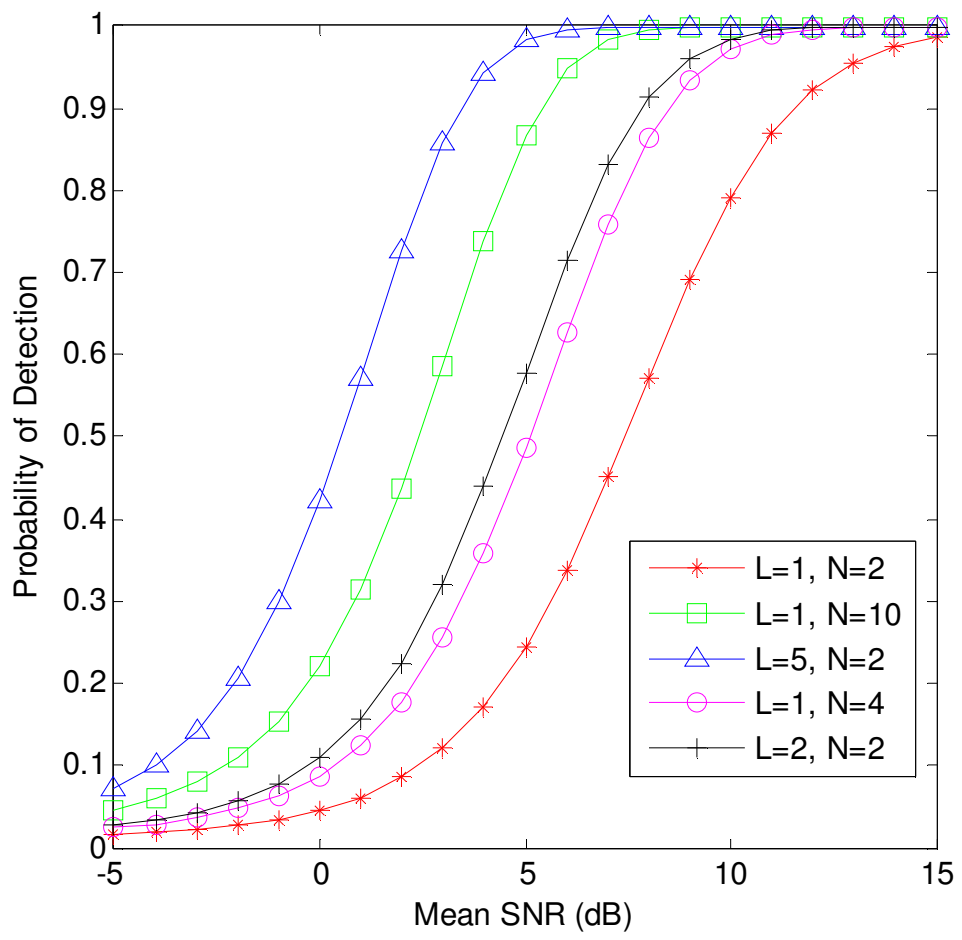

Fig. 8 Performance of wideband sensing using peak detectors with MRC diversity over a Rice $(K=3)$ multipath fading channel, with various $L$ and $N$, total $u=10$ and $P_{f}=0.01$. 


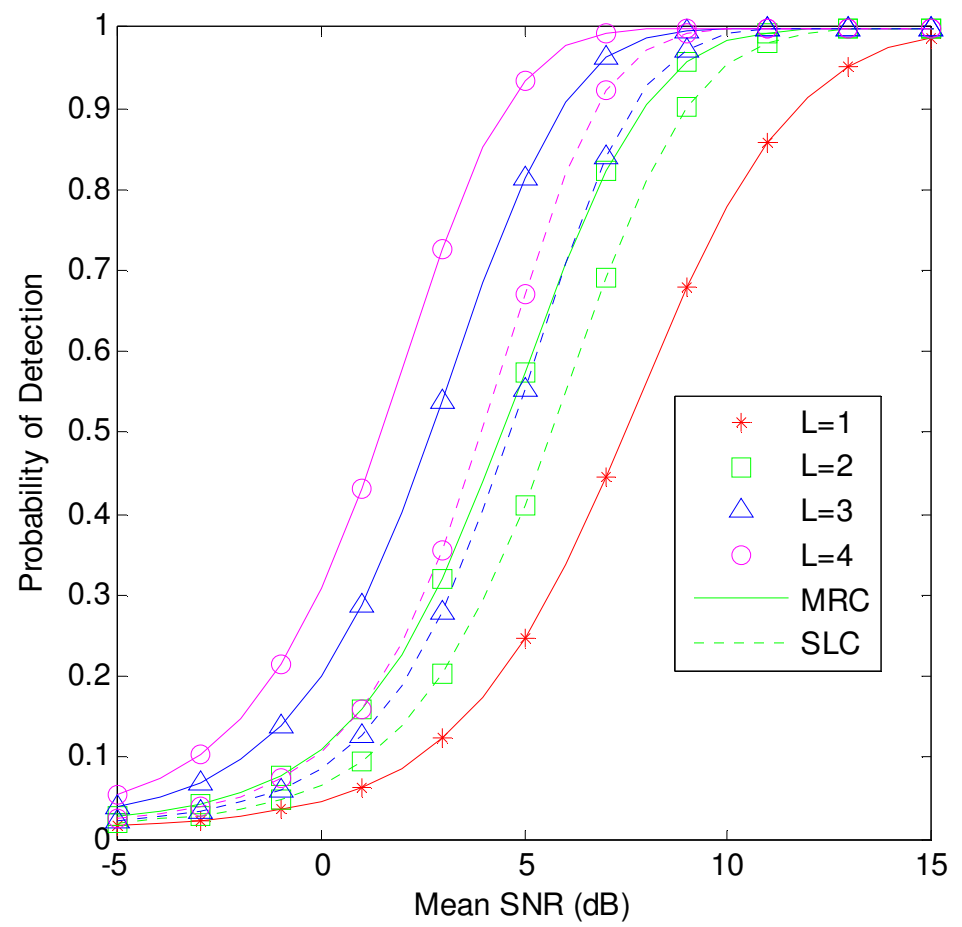

Fig. 9 Performance of wideband sensing using 2-NB peak detector with MRC and SLC diversity over a Nakagami-m $(m=2)$ multipath fading channel, with $L=\{1,2,3,4\}$, total $u=$ 10 , and $P_{f}=0.01$.

\section{Conclusions}

In this article, we have considered the extension of traditional energy detector to wideband sensing with improved performance. The solution involves the use of parallel detection circuitry with each tuned to different frequencies. This introduces some resolution component and improved sensitivity based on the number of parallel circuits and bandwidth range of each circuit. Since the bandwidth of the incumbent signal is generally not known a priori, there is possibility that it spans across multiple detectors. The detection of such wideband signal can be achieved by either data or decision fusion of the results of each narrowband detector. We show that the use of parallel multi-channel detection can greatly enhance the detection of wideband signal compared to averaging while improving the resolution and sensitivity of energy detection. Furthermore, we show that the number of required NB detectors can be greatly reduced with diversity detection. In fact, the $N L$ product can be used to achieve various design configurations with different degrees of implementation complexity. To the best of our knowledge, the application of energy detection in conjunction with data/decision fusion strategies and diversity reception reported in this article in the context of wideband heterogeneous spectrum sensing is new and has not been reported in the literature (i.e., the data/decision fusion in this article is fundamentally different from the conventional cooperative spectrum sensing literature that focuses on improving the detection probability of a NB signal through user cooperation or distributed spatial-diversity because there is no reporting/transmission errors and our objective of data/decision fusion is focused on improving the detection performance of wideband PU signals that could span over multiple NB energy detectors). 
International Journal of Computer Networks \& Communications (IJCNC) Vol.4, No.3, May 2012

\section{APPENDix A}

The kth order derivates of MGF of SNR of common fading channels are provided in this section.

\section{Rayleigh Fading Channel}

Rayleigh distribution is used to model the variation of the amplitude of received signal in a multipath fading with no direct line-of-sight (LOS) path. The received SNR (square of fade amplitude) can be modelled according to exponential distribution and it is given by [24, Table 2.2]

$$
f_{\gamma}(\gamma)=\frac{1}{\Omega} e^{-\gamma / \Omega}
$$

where $\Omega=E[\gamma]$ is the expectation or average of instantaneous received SNR.

The corresponding MGF expression can be evaluated by taking the Laplace transform of the $\mathrm{PDF}$ and it is given by

$$
\phi_{\gamma}(s)=\int_{0}^{\infty} e^{-s \gamma} \frac{1}{\Omega} e^{-\gamma / \Omega} d \gamma=(1+s \Omega)^{-1}
$$

The kth order derivative with respect to (w.r.t) the Laplace variable s can be expressed as

$$
\phi_{\gamma}^{(k)}(s)=\frac{(-\Omega)^{k} \Gamma(1+k)}{(1+s \Omega)^{1+k}}
$$

For an $L$-branch MRC diversity system with independent and identically distributed statistics, the effective MGF is given by

$$
\phi_{\gamma}(s)=(1+s \Omega)^{-L}
$$

The corresponding $k$ th order derivative with respect to (w.r.t) the Laplace variable $s$ can be expressed as

$$
\phi_{\gamma}^{(k)}(s)=\frac{(-\Omega)^{k} \Gamma(L+k)}{(1+s \Omega)^{L+k} \Gamma(L)}
$$

\section{Rice Fading Channel}

The Rice distribution also known as Nakagami- $n$ distribution is used to model the variation of the amplitude of received signal in a multipath fading consisting of one strong LOS component path. The fade distribution is often used to model the propagation of radio waves in satellite communication and other LOS terrestrial wireless transmission backbone. The received signal SNR (square of fade amplitude) can be modelled according to non-central chi-square distribution and it is given by [23, Table 2.2]

$$
\frac{1+K}{\Omega} \exp \left(-K-\frac{(1+K) \gamma}{\Omega}\right) I_{o}(2 \sqrt{K(1+K) \gamma / \Omega})
$$

where $K$ is the ratio of the power of LOS (specular) component to the average of the power of the scattered component otherwise known as the Rice factor. 
International Journal of Computer Networks \& Communications (IJCNC) Vol.4, No.3, May 2012

The corresponding MGF expression can be evaluated similar to the case of Rayleigh fading above and is given by

$$
\phi_{\gamma}(s)=\left(\frac{1+K}{1+K+s \Omega}\right) \exp \left(\frac{-K s \Omega}{1+K+s \Omega}\right)
$$

The $k$ th order derivative after few algebraic manipulations can then be expressed as

$$
\phi_{\gamma}^{(k)}(s)=\frac{(-\Omega)^{k}(k !)^{2}(1+K)}{(1+K+s \Omega)^{1+k}} \exp \left(\frac{-s K \Omega}{1+K+s \Omega}\right) \sum_{i=0}^{k} \frac{1}{(i !)^{2}(k-1) !}\left(\frac{K(1+K)}{1+K+s \Omega}\right)^{i}
$$

For an $L$-branch MRC diversity system with independent and identically distributed statistics, the effective MGF is given by

$$
\phi_{\gamma}(s)=\left(\frac{1+K}{1+K+s \Omega}\right)^{L} \exp \left(\frac{-K L s \Omega}{1+K+s \Omega}\right)
$$

The corresponding $k$ th order derivative with respect to (w.r.t) the Laplace variable $s$ can be expressed as

$$
\phi_{\gamma}^{(k)}(s)=\frac{(-\Omega)^{k} k !(L+k-1) !(1+K)^{L}}{(1+K+s \Omega)^{L+k}} \exp \left(\frac{-s K L \Omega}{1+K+s \Omega}\right) \sum_{i=0}^{k} \frac{1}{i !(L+i-1) !(k-i) !}\left(\frac{K L(1+K)}{1+K+s \Omega}\right)^{i}
$$

\section{Nakagami-m Fading Channel}

Nakagami-m distribution follows a central chi-square distribution and was originally developed as an approximation of Rice distribution as it is much easier to handle compare to the Rice distribution. The distribution has been adopted in the analysis of many terrestrial wireless communications system. The received signal SNR (square of fade amplitude) can be modelled according to gamma distribution and it is given by [24, Table 2.2]

$$
f_{\gamma}(\gamma)=\left(\frac{m}{\Omega}\right)^{m} \frac{\gamma^{m-1} e^{-\frac{m}{\Omega} \gamma}}{\Gamma(m)}
$$

where $\Omega=\mathrm{E}[\gamma]$ is the expectation or average of instantaneous received SNR and $m$ is the Nakagami-m fading severity index.

The corresponding MGF expression can be evaluated by taking the Laplace transform of the $\mathrm{PDF}$ and is given by

$$
\phi_{\gamma}(s)=\int_{0}^{\infty} e^{-s \gamma}\left(\frac{m}{\Omega}\right)^{m} \frac{\gamma^{m-1} e^{-\frac{m}{\Omega} \gamma}}{\Gamma(m)} d \gamma=(1+s \Omega / m)^{-m}
$$

The $k$ th order derivative w.r.t the Laplace variable $s$ can be expressed as

$$
\phi_{\gamma}^{(k)}(s)=\frac{(-\Omega)^{k} m^{m} \Gamma(m+k)}{(m+s \Omega)^{m+k} \Gamma(m)}
$$

For an $L$-branch MRC diversity system with independent and identically distributed statistics, the effective MGF is given by

$$
\phi_{\gamma}(s)=(1+s \Omega / m)^{-m L}
$$

The corresponding $k$ th order derivative with respect to (w.r.t) the Laplace variable $s$ can be expressed as 
International Journal of Computer Networks \& Communications (IJCNC) Vol.4, No.3, May 2012

$$
\phi_{\gamma}^{(k)}(s)=\frac{(-\Omega)^{k} m^{m L} \Gamma(m L+k)}{(m+s \Omega)^{m L+k} \Gamma(m L)}
$$

\section{Weibull Fading Channel}

This also one of the important probability models normally associated with multipath in mobile radio system operating at frequency range of $800 / 900 \mathrm{MHz}$ [24]. The PDF of the received signal SNR is given by [24, (2.29)]

$$
f_{\gamma}(\gamma)=\frac{c}{2}\left(\frac{\Gamma\left(1+\frac{2}{c}\right)}{\Omega}\right)^{\frac{c}{2}} \gamma^{c / 2-1} \exp \left[\left(-\left(\frac{\gamma}{\Omega}\right) \Gamma\left(1+\frac{2}{c}\right)\right)^{\frac{c}{2}}\right]
$$

where $c$ is a shape parameter that is chosen for best fit to measurement results and it gives the shape flexibility of the Nakagami-m distribution.

The corresponding MGF expression can be evaluated by taking the Laplace transform of the PDF and is given by

$$
\phi_{\gamma}(s)=\left(\frac{\Gamma\left(1+\frac{2}{c}\right)}{\Omega}\right)^{\frac{c}{2}}(2 \pi)^{(1-c / 2) / 2}\left(\frac{c}{2}\right)^{\frac{c}{2}+\frac{1}{2}} s^{-\left(\frac{c}{2}+1\right)} G_{1, c / 2}^{c / 2,1}\left(\left(\frac{2 s \Gamma\left(1+\frac{2}{c}\right)}{c \Omega}\right)^{-\frac{c}{2}} \mid 1,1+\frac{2}{c}, \ldots, 2+\frac{-2}{c}\right)
$$

where G(.) is the Meijer's G function defined by [25, Eq. (9.3)].

The $k$ th order derivative with respect to w.r.t the Laplace variable $s$ can then be expressed as

$$
\phi_{\gamma}^{(k)}(s)=\left(\frac{\Gamma\left(1+\frac{2}{c}\right)}{\Omega}\right)^{\frac{c}{2}}(2 \pi)^{(1-c / 2) / 2}\left(\frac{c}{2}\right)^{k+\frac{c}{2}+\frac{1}{2}} s^{-\left(k+\frac{c}{2}+1\right)} G_{1, c / 2}^{c / 2,1}\left(\left(\frac{2 s \Gamma\left(1+\frac{2}{c}\right)}{c \Omega}\right)^{-\frac{c}{2}} \mid 1+\frac{2 k}{c}, 1+\frac{2(k+1)}{c}, \ldots, 2+\frac{2(k-1)}{c}\right)
$$

\section{ACKNOWLEDGEMENTS}

This work is supported in part by funding from the US Army Research Office (W911NF-04-20054), Clarkson Aerospace, and National Science Foundation (0931679 \& 1040207).

\section{REFERENCES}

[1] "IEEE standard definitions and concepts for dynamic spectrum access: Terminology relating to emerging wireless networks, system functionality, and spectrum management," IEEE Std 1900.12008, pp. c1-48, 262008.

[2] S. Chan, "Shared spectrum access for the DoD," in New Frontiers in Dynamic Spectrum Access Networks, Proc. 2nd IEEE International Symposium on Dynamic Spectrum Access, DySPAN 2007, pp. $524-534$, April 2007.

[3] E. Hossain, D. Niyato, and Z. Han, Dynamic Spectrum Access and Management in Cognitive Radio Networks. Cambridge, UK: Cambridge University Press, 2009.

[4] K. Chowdhury and I. Akyildiz, "Cognitive Wireless Mesh Networks with Dynamic Spectrum Access," IEEE Journal on Selected Areas in Communications, vol. 26, no. 1, pp. 168 -181, Jan. 2008. 
International Journal of Computer Networks \& Communications (IJCNC) Vol.4, No.3, May 2012

[5] Q. Zhao and B. Sadler, "A Survey of Dynamic Spectrum Access," Signal Processing Magazine, IEEE, vol. 24, no. 3, pp. 79-89, May 2007.

[6] S. Haykin, "Cognitive radio: Brain-empowered Wireless Communications," IEEE Journal on Selected Areas in Communications, vol. 23, no. 2, pp. 201 - 220, Feb. 2005.

[7] J. Boksiner and S. Dehnie, "Cognitive Comparison of Energy Detection using Averaging and Maximum Values Detection for Dynamic Spectrum Access," 34th IEEE Sarnoff Symposium, 2011, pp. 1-6, May, 2011.

[8] H. Urkowitz, "Energy detection of Unknown deterministic Signals," Proc IEEE, vol. 55, no. 4, pp. 523-531, Apr 1967.

[9] A. Annamalai, O. Olabiyi, S. Alam, O. Odejide, and D. Vaman, "Unified Analysis of Energy Detection of Unknown Signals over Generalized Fading Channels," Proc. IEEE IWCMC'11 Conf., Turkey, Sept, 2011.

[10] O. Olabiyi, and A. Annamalai, "Further Results on the Performance of Energy Detector over Generalized Fading Channels," Proc. IEEE PIMRC Conf. 2011, Toronto, Canada, pp 489-493, Sept., 2011.

[11]O. Olabiyi, S. Alam, O. Odejide, and A. Annamalai, "On the Energy Detection of Unknown Deterministic Signals over Generalized Fading Channel,” Proc. IWCN Conf. 2011, Las Vegas, USA, July, 2011.

[12]Fadel F. Digham, Mohamed-Slim Alouni and Marvin K. Simon, "On the Energy Detection of Unknown Signals Over Fading Channels," IEEE Trans. on Commununications, vol 55, no.1, pp.2124, Jan. 2007.

[13] S. Herath, N. Rajatheva, C. Tellambura, "Unified Approach for Energy Detection of Unknown Deterministic Signal in Cognitive Radio over Fading Channels” Proc. IEEE ICC'09, June 2009, Germany.

[14]H. Li, C. Li and H. Dai," Quickest Spectrum Sensing in Cognitive Radio,” Proc. CISS 2008, pp.203208.

[15]L. Lai, Y. Fan, and H.V. Poor,"Quickest Detection in Cognitive Radio: A Sequential Change Detection Framework," Proc. IEEE GLOBECOM 2008, pp.1-5.

[16] S. Zarrin and T.J. Lim,"Cooperative Quickest Spectrum Sensing in Cognitive Radios with Unknown Parameters," Proc. IEEE GLOBECOM 2009, June 2009, pp.1-6.

[17] Q. Zhao and J. Ye, "Quickest Detection in Multiple On-Off Processes," IEEE Trans. on Signal Processing, vol.58, no.12, pp.5994-6006, Dec. 2010.

[18]J. C. K. Liang and S. D. Blostein, " Reduced Complexity Multiband Multi-Sensor Spectrum Sensing," Proc. IEEE PIMRC 2011, pp.320-325, Sept. 2011.

[19] W. Zhang and K. B. Letaief, "Cooperative spectrum sensing with transmit and relay diversity in cognitive radio networks," IEEE Transactions on Wireless Communications, vol. 7, no. 12, pp. 4761-4766, Dec. 2008.

[20] S. Atapattu, C. Tellambura, H. Jiang, "Energy Detection Based Cooperative Spectrum Sensing in Cognitive Radio Networks," IEEE Transactions on Wireless Communications,, vol.PP, no.99, pp.1$10,2011$.

[21] O. Olabiyi, and A. Annamalai, "Analysis of Cooperative Relay-Based Energy Detection of Unknown Deterministic Signals in Cognitive Radio Networks," Proc. of IWCN Conf. 2011, Las Vegas, USA, July, 2011.

[22] O. Olabiyi, and A. Annamalai, "Performance Evaluation of Cooperative Cognitive Radio Networks with Data/Decision Fusion," to appear in the Proc. IEEE MILCOM Conference 2011, Baltimore, USA, Nov. 2011.

[23] G. M. Dillard, "Recursive Computation of the Generalized Q-function," IEEE Trans. Aero. Elec. Sys., vol. AES-9, pp. 614-615, July 1973. 
International Journal of Computer Networks \& Communications (IJCNC) Vol.4, No.3, May 2012

[24] M. K. Simon and M-S Alouini, Digital Communication over Fading Channels, New York: Wiley, 2 edition, 2005.

[25] Gradshteyn, I. S. and Ryzhik, I. M. (1994) Table of Integrals, Series and Products, 5th ed., San Diego, CA: Academic.

Oluwatobi O. Olabiyi received the B.Sc. degree in Electronic and Electrical Engineering from Obafemi Awolowo University, Ile-Ife, Nigeria, and the M.S. degree in Electrical Engineering Prairie View A\&M University, Texas, USA. Over the last two years, he has co-authored approximately two-dozen peer-reviewed conference and journal articles. He was the recipient of the Roy G. Perry College of Engineering Outstanding Masters Student of the Year Award (2011) and the National Society of Black Engineer's Golden Torch Award for Graduate Student of Year (2012). He is presently continuing his doctoral studies at the Prairie View A\&M University. His research interests include dynamic spectrum access, MIMO, cooperative communications, statistical signal processing, compressive sensing, machine-learning and optimization techniques.

Dr. Annamalai is presently the Director of Center of Excellence for Communication Systems Technology Research, a Texas A\&M Board of Regents approved University Research Center at the Prairie View A\&M University, and a tenured faculty member in the Department of Electrical and Computer Engineering. He has over 16 years of research/teaching experience in wireless communications at Motorola, University of Victoria, Air Force Research Laboratory, Virginia Tech and PVAMU with approximately 200 peer-reviewed publications and 5 book chapters. Dr. Annamalai has been honored by his colleagues on numerous occasions for his excellence in research including winning the 2011 Roy G. Perry College of Engineering Outstanding Faculty (Research) Award, IEEE Leon Kirchmayer Prize Paper award, ASEE/AFOSR Summer

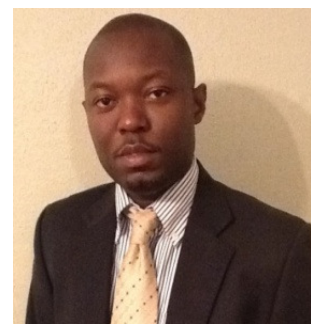
Faculty Fellowships, NSERC Doctoral Prize, CAGS/UMI Distinguished Doctoral Dissertation Award, IEEE VTS/Motorola Daniel E. Noble Fellowship, among others. He had served on the Editorial Boards of four IEEE journals/transactions in the last 12 years, and has helped to organize a few major IEEE conferences on wireless communications including serving in the capacity of Technical Program Chair of the 2002 IEEE Vehicular Technology Conference in Vancouver, Canada. His current research interests include cooperative spectrum sensing, compressive sensing, cross-layer design for scalable multimedia transmission and cooperative wireless communications. 